\title{
Saccular portal vein aneurysm complicating laparoscopic cholecystectomy
}

\author{
Haider Rasheed ${ }^{1}$, Mohammed Abed ${ }^{2}$, and Duraid Mahmoud Jamil ${ }^{1}$
}

${ }^{1} \mathrm{Al}$ Yarmouk Teaching Hospital

${ }^{2}$ College of Medicine Ibn Sina University of Medical and Pharmaceutical Sciences

November 20, 2021

\begin{abstract}
Portal vein aneurysm (PVA) is a rare vascular entity of uncertain etiology. Saccular PVA is the less frequently reported morphology but often with more symptoms or complications. Ultrasound, along with color doppler study, is a valuable tool in the diagnosis and follow-up.
\end{abstract}

Full title: Saccular portal vein aneurysm complicating laparoscopic cholecystectomy

Running head: Saccular portal vein aneurysm

Haider Rasheed

Radiology resident, Al Yarmuk Teaching Hospital, Baghdad, 10001 Iraq haidersaeed.90@gmail.com

ORCID ID: https://orcid.org/0000-0002-6280-4149

Mohammed Abed

College of Medicine, Ibn Sina University of Medical and Pharmaceutical Sciences, Baghdad,10001 Iraq mohammed.abed@outlook.sa

ORCID ID: https://orcid.org/0000-0002-2210-034X

\section{Duraid Mahmoud Jamil}

Radiologist, Al Yarmuk Teaching Hospital, Baghdad, 10001 Iraq

FIBMS (fellow of Iraqi Board of medical Specialization of diagnostic radiology)

EDIR (European diploma in radiology)

ORCID ID: https://orcid.org/0000-0003-1764-1288

duraid_mahmoud@yahoo.com

*Corresponding author

\section{Mohammed Abed}

Ibn Sina University of Medical and Pharmaceutical Sciences, Baghdad,10001 Iraq

mohammed.abed@outlook.sa

Current address: Baghdad, 10001 IRAQ 
009647728259802

\section{Patient Consent}

Written informed consent was obtained from the patient to publish this report in accordance with the journal's patient consent policy.

\section{Key messages:}

Portal vein aneurysm, the rare saccular type, is associated with more complications than the fusiform one.

Once diagnosed, PVA shall be followed up periodically by ultrasound.

\section{ABSTRACT}

Portal vein aneurysm (PVA) is a rare vascular entity of uncertain etiology. Saccular PVA is the less frequently reported morphology but often with more symptoms or complications. Ultrasound, along with color doppler study, is a valuable tool in the diagnosis and follow-up, but a CT scan with contrast can best delineate the aneurysm relative to the adjacent structures. We present a case of saccular PVA complicating a laparoscopic cholecystectomy.

Keywords

Cholecystectomy, Laparoscopic; Aneurysm; Portal Vein; Ultrasonography, Doppler; computerized tomography

\section{Case Report}

A 39-year-old female was referred to our outpatient ultrasound unit for intermittent right upper quadrant abdominal pain, unrelated to meals, often resolved spontaneously, associated with nausea without vomiting, jaundice, weight loss, or change in bowel habits. Physical exam was only remarkable for mild right upper quadrant tenderness. Murphey's sign was negative, and there was no organomegaly. Initial lab tests were unremarkable. Two years ago, she had Laparoscopic cholecystectomy for calculus cholecystitis, documented by ultrasound scan that showed an increased wall thickness and multiple stones with positive probe tenderness. The postoperative course was uneventful.

The patient was scanned with GE Voluson E6 device (GE Healthcare Chicago, IL, USA) using curvilinear $(3 \mathrm{~Hz})$ probe and showed a cystic lesion in close proximity to the porta-hepatis measured about $3.5 \mathrm{X} 3.3$ $\mathrm{cm}$ (figure 1). Further evaluation with color doppler study demonstrated a venous flow pattern that was unidirectional on rest and bidirectional with expiration(figure 2); features were suspicious of an extrahepatic portal vein aneurysm (video 1). A contrast-enhanced CT scan of the upper abdomen was then ordered.

The CE-CT scan was done using PHILIPS Brilliance iCT 128 CT Scanner (Philips Healthcare, USA). The portal phase showed a well-defined saccular aneurysm in the proximal segment of the portal vein near the confluence without luminal filling defects. The sac was measuring about $3.5 \mathrm{X} 3.3 \mathrm{~cm}$, with the neck of 1.7 $\mathrm{cm}$, exerting mass effect in the form of downward displacement of the pancreatic head (figure 3 ) and pressure effect on the duodenum. The distal extrahepatic portion and intrahepatic segment were normal, and the splenic vein and superior mesenteric vein were normal as well. The pancreatic parenchyma was normal, without surrounding fat stranding or collection. The gall bladder bed was clear. Patient was then referred to an interventional radiologist for closure of the neck of the aneurysmal sac.

\section{Discussion}

PVA is a rare entity incidentally discovered and of unclear etiology. In our case, the patient was presented with persistent right upper quadrant discomfort two years after she has undergone a laparoscopic cholecystectomy. In the previous two studies ${ }^{1,2}$, the occurrence of PVA post-cholecystectomy was ten and forty years after, compared to two years only in our case. We suggest that degenerative changes might occur after the operation that supports the acquired theory already proposed in a previous study ${ }^{3}$ because there was no portal hypertension in our case. The timing difference may be related to the operation itself or any 
associated mild trauma to the portal vein at the time of surgery. The close proximity of the aneurysm in our case to the surgical clips supports the iatrogenic theory of PVA.

The ultrasound with doppler study offers cheap and radiation-free assessment and follow-up for such possible complications. It can also evaluate the patency of the portal vein and the hemodynamic flow. However, a computed CT scan can best assess the exact location of the aneurysm and guide any future intervention. Although the diagnosis can be made without contrast, our case showed a substantial advantage of the use of contrast-enhanced CT scan since it allows accurate delineation of the aneurysm from the adjacent structures such as bowel or lymph nodes. It can also demonstrate any associated portomesentric thrombus, which can alter the management plan.

Saccular PVA, as in our case, is the less common variety than fusiform but often associated with more complications such as rupture or pressure effects. In the previous studies ${ }^{1,2,4}$, a post-cholecystectomy PVA was originally associated with gall stones mandated the removal of the gall bladder. Similarly, these patients at risk for gallstone pancreatitis, which could also contribute to developing PVA as an important risk factor ${ }^{5}$. Another possible explanation of the patient's present complaint is the pressure effect from the aneurysm itself to the head of the pancreas that resulted in a mild form of chemical pancreatitis or pressure on the sphincter of Oddi, producing what has been described as a postcholecystectomy syndrome ${ }^{6}$

Since the management options of PVA is still controversial ${ }^{7}$, in our case, the persistent symptoms post laparoscopic cholecystectomy for a stone disease should raise the possibility of PVA, and when diagnosed, a periodic assessment by ultrasound is recommended to allow the surgeons to intervene at the appropriate time.

\section{Conflict of Interest}

Authors declare they have no competing interests.

\section{Acknowledgments}

Declared none.

\section{Contributors}

Haider Rasheed \& Duraid Mahmoud Jamil contributed by the pictures, editing and revision of the article. Mohammed Abed drafted the article and contributed to editing and revision.

All authors approved submission of the manuscript.

\section{Patient Consent}

Obtained.

\section{References:}

1. Guilbaud T, Birnbaum DJ, Duconseil P, Soussan J, Moutardier V. Portal vein aneurysm incidentaloma. Surgery. 2017;162(5):1177-8.

2. Flis V, Gadžijev EJH. Reconstruction of the main portal vein for a large saccular aneurysm. 2003;5(3):18890 .

3. Ohnishi K, Nakayama T, Saito M, Nomura F, Koen H, Tamaru J, et al. Aneurysm of the intrahepatic branch of the portal vein: report of two cases. 1984;86(1):169-73.

4. Ozbek SS, Killi MR, Pourbagher MA, Parildar M, Katranci N, Solak A. Portal venous system aneurysms: report of five cases. Journal of ultrasound in medicine : official journal of the American Institute of Ultrasound in Medicine. 1999;18(6):417-22; quiz 23.

5. Dalal PS, Raman SP, Horton KM, Fishman EK. Portal vein aneurysms: imaging manifestations and clinical significance. Emergency radiology. 2013;20(5):453-7. 
6. Zackria R, Lopez RA. Postcholecystectomy Syndrome. StatPearls. Treasure Island (FL): StatPearls Publishing Copyright (C) 2021, StatPearls Publishing LLC.; 2021.

7. Watanabe Y, Takase K, Okada K, Aikawa M, Okamoto K, Koyama I. Portal vein aneurysm with complete spontaneous regression after 10 years using conservative treatment. Clinical journal of gastroenterology. 2020;13(5):940-5.

Figure 1: Echo-free cystic lesion at the right upper quadrant

Figure 2: Spectral analysis demonstrate the flow pattern.

Figure 3: CE-CT scan portal phase demonstrate a well-defined saccular aneurysm in the proximal segment of the portal vein exerting pressure on the pancreas and duodenum.

Video 1: Color doppler study demonstrated a venous flow pattern that was unidirectional on rest and bidirectional with expiration.

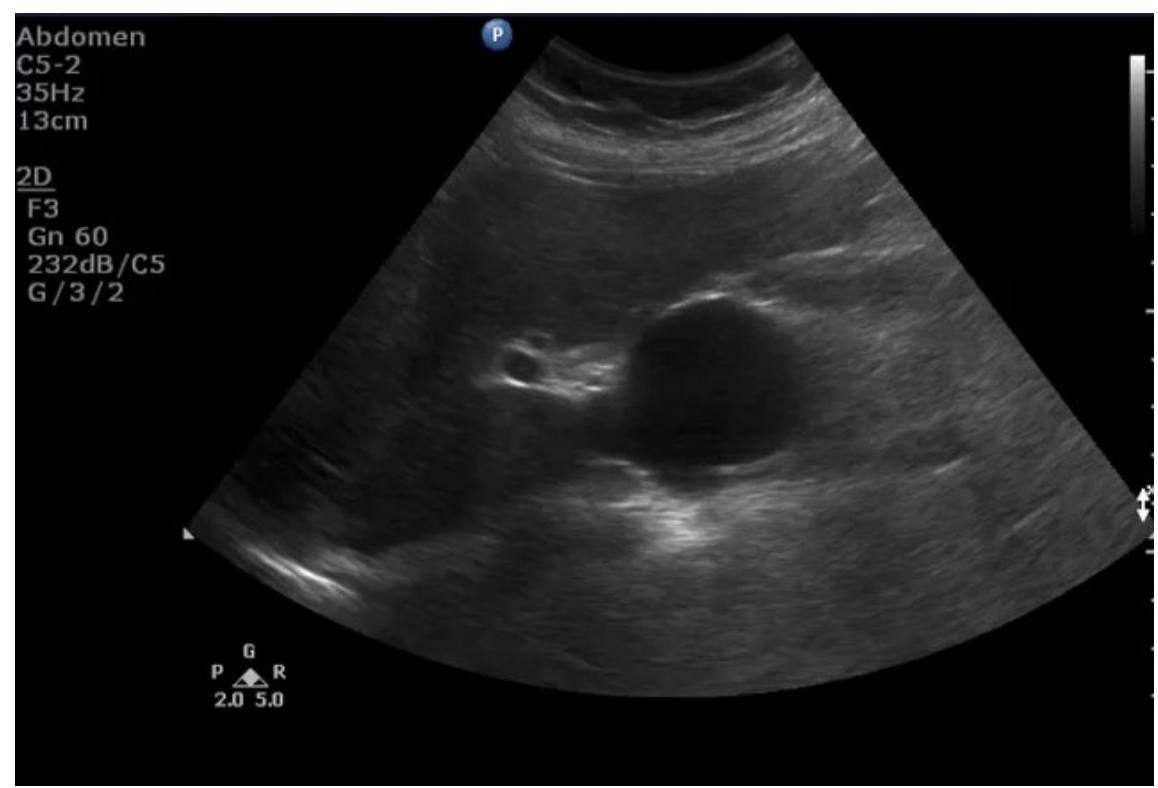



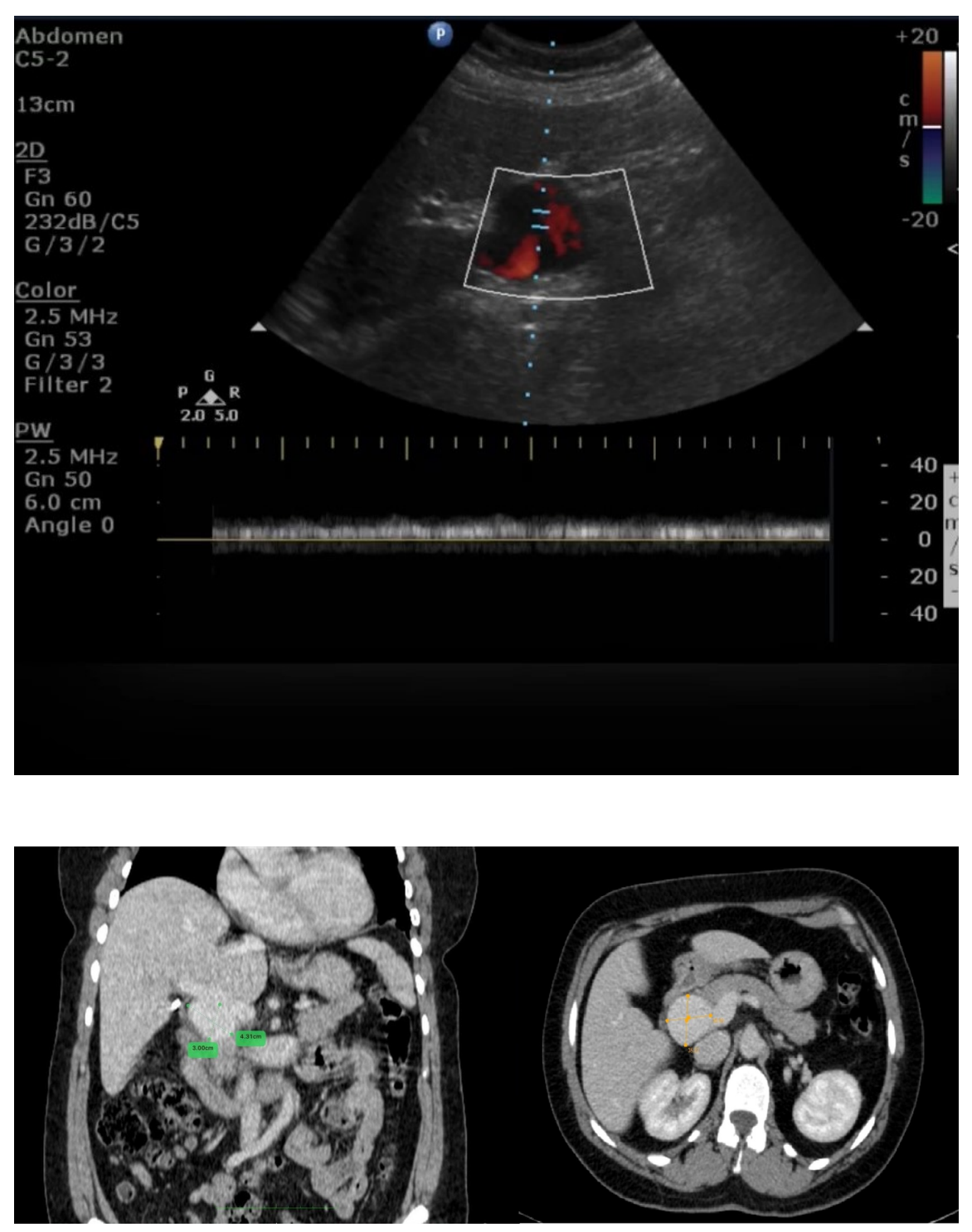